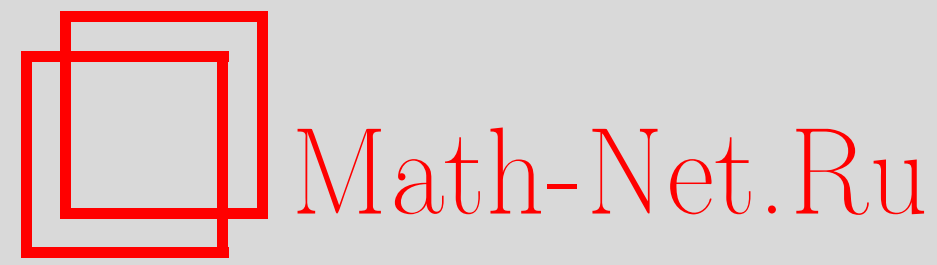

Ш. А. Хашимов, Г. Р. Абдурахманов, Об оценке скорости сходимости в центральной предельной теореме для обобщенных $U$-статистик, Теория вероятн. и ее примен., 1998, том 43, выпуск 1, 69-81

DOI: https://doi.org/10.4213/tvp824

Использование Общероссийского математического портала Math-Net.Ru подразумевает, что вы прочитали и согласны с пользовательским соглашением http://www . mathnet.ru/rus/agreement

Параметры загрузки:

IP : 54.224 .187 .69

26 апреля 2023 г., 13:50:10

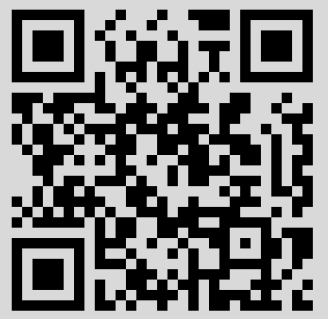


(c) 1998 г. ХАШИМОВ ШІ. А., АБДУРАХМАНОВ Г. Р.

\section{ОБ ОЩЕНКЕ СКОРОСТИ СХОДИМОСТИ В ЦЕНТРАЛЬНОЙ ПРЕДЕЛЬНОЙ ТЕОРЕМЕ ДЛЯ ОБОБЩЕННЫХ $U$-СТАТИСТИК}

В статье устанавливаются оценки скорости сходимости в центральной предельной теореме для обобшенных невырожденных $U$-статистик. Полученная оценка уточняет и обобщает известные ранее результаты в этом направлении.

Ключевые слова и фразы: обобщенная $U$-статистика, характеристическая функция, нормальное распределение.

Пусть $X_{1}, \ldots, X_{n}$ - последовательность независимых случайных величин со значениями в каком-либо измеримом пространстве $\mathscr{X}$.

Рассмотрим последовательность статистик

$$
U_{n}=\frac{1}{n(n-1)} \sum_{1 \leqslant i \neq j \leqslant n} h_{i j}\left(X_{i}, X_{j}\right), \quad n \geqslant 2,
$$

образованных семейством борелевских функций $\left\{h_{i j}(x, \dot{y}), x, y \in \mathscr{X}, i, j=\right.$ $1, \ldots, n, i \neq j\}$, предполагая, что последние могут зависеть от $n$ (и обязательно зависят от $n$, если они одинаковы при всех $i \neq j, i, j=1, \ldots, n)$, a распределения величин $X_{i}$ могут зависеть от $i$ и $n$.

Пусть, далее, для всех $i \neq j, n \geqslant 2$,

$$
\begin{array}{ll}
h_{i j}(x, y)=h_{j i}(y, x), & x, y \in \mathscr{X}, \\
\mathbf{E}\left|h_{i j}\left(X_{i}, X_{j}\right)\right|<\infty, & \mathbf{E} h_{i j}\left(X_{i}, X_{j}\right)=0 .
\end{array}
$$

Статистики вида (1) назовем обобщенными $U$-статистиками (степени 2), а функции $h_{i j}(x, y)$ - их ядрами. Для классической модели $U$-статистик (впервые подробно рассмотренных в [1]), порожденных одной бесконечной последовательностью независимых величин $X_{1}, X_{2}, \ldots$ и одним фиксированным симметричным ядром $h(x, y)$, известны аналоги многих предельных теорем для сумм независимых случайных величин.

В частности, в работе [1] при условии $\mathrm{E} h^{2}\left(X_{1}, \dot{X}_{2}\right)<\infty$ в случае, когда $X_{1}, X_{2}, \ldots$ одинаково распределены и ядро невырождено, т.е.

$$
\operatorname{DE}\left[h\left(X_{1}, X_{2}\right) \mid X_{1}\right] \neq 0,
$$

*Институт математики АН РУ, ул. Ф. Ходжаева, 29, 700143 Ташкент, Узбекистан. 
установлена центральная предельная теорема (ц.п.т.) для $U$-статистик. Там же получена ц.п.т. и для $U$-статистик от различно распределенных величин. Позднее ц.п.т. была доказана при условии (2) и условии конечности левой части (2) и момента $\mathbf{E}\left|h\left(X_{1}, X_{2}\right)\right|^{4 / 3}$ (см. $[2$, теоремы 1 и 4.2 .2$])$.

Далее на протяжении многих лет рядом авторов с той или иной степенью точности и при различных моментных условиях оценивалась скорость сходимости в ц.п.т. для классической модели $U$-статистик степени $r \geqslant 2$ (см., например, работы [3]-[7], а также [2]).

В случае обобщенной модели $U$-статистик со степенью $r \geqslant 2$ предельные распределения изучались в работах [8]-[12]. В работе [13] получена равномерная оценка в ц.п.т. при условии

$$
\mathbf{E} h_{i j}^{2}\left(X_{i}, X_{j}\right)<\infty \quad \text { для всех } i \neq j
$$

в случае, когда хотя бы одно ядро $h_{i j}(x, y)$ является невырожденным, т.е. для него верно условие типа (2).

В работе [11] была доказана асимптотическая нормальность обобшенных $U$-статистик при более слабом требовании, чем (3):

$$
\sup _{n \geqslant 2} \max _{1 \leqslant i<j \leqslant n} \mathbf{E}\left|h_{i j}\left(X_{i}, X_{j}\right)\right|^{4 / 3} I\left(\left|h_{i j}\left(X_{i}, X_{j}\right)\right|>\rho\right) \longrightarrow 0 \quad \text { при } \rho \rightarrow \infty
$$

Настоящая работа посвящена установлению равномерной ощенки скорости сходимости в ц.п.т. для обобщенных $U$-статистик при нарушении условия (3). Полученная здесь теорема уточняет основной результат работы [11].

Введем обозначения

$$
\begin{aligned}
& \left.\begin{array}{c}
\Psi_{i j}(x)=\mathbf{E}\left[h_{i j}\left(X_{i}, X_{j}\right) \mid X_{i}=x\right], \\
U_{n 1}=\frac{1}{n} \sum_{i=1}^{n} \widetilde{h}_{i}\left(X_{i}\right), \quad \widetilde{h}_{i}(x)=\frac{1}{n-1} \sum_{\substack{j=1, \ldots, n \\
j \neq i}} \Psi_{i j}(x),
\end{array}\right\} \\
& \left.\begin{array}{c}
U_{n 2}=\frac{1}{n(n-1)} \sum_{1 \leqslant i \neq j \leqslant n} \tilde{h}_{i j}\left(X_{i}, X_{j}\right), \\
\widetilde{h}_{i j}(x, y)=h_{i j}(x, y)-\Psi_{i j}(x)-\Psi_{i j}(y) .
\end{array}\right\}
\end{aligned}
$$

Положим

$$
\left.\begin{array}{ll}
\beta_{i n}^{2}=\mathbf{E} \widetilde{h}_{i}^{2}\left(X_{i}\right)=\mathbf{D} \widetilde{h}_{i}\left(X_{i}\right), & \sigma_{m}^{2}=\sum_{i=1}^{m} \beta_{i n}^{2}, \\
\beta_{n}^{2}=\min _{1 \leqslant i \leqslant n} \beta_{i n}^{2}, & \beta^{2}=\inf _{n \geqslant 2} \beta_{n}^{2} .
\end{array}\right\}
$$

Обозначим также

$$
\left.\begin{array}{ll}
\mu_{p}^{(n)}=\max _{1 \leqslant i \neq j \leqslant n} \mathbf{E}\left|h_{i j}\left(X_{i}, X_{j}\right)\right|^{p}, & \mu_{p}=\sup _{n \geqslant 2} \mu_{p}^{(n)}, \\
\nu_{q}^{(n)}=\max _{1 \leqslant i \neq j \leqslant n} \mathbf{E}\left|\Psi_{i j}\left(X_{i}\right)\right|^{q}, & \nu_{q}=\sup _{n \geqslant 2} \nu_{q}^{(n)},
\end{array}\right\}
$$




$$
B_{p}^{(n)}=\frac{\left[\mu_{p}^{(n)}\right]^{1 / p}}{\beta_{n}}, \quad \Gamma_{q}^{(n)}=\frac{\left[\nu_{q}^{(n)}\right]^{1 / q}}{\beta_{n}}
$$

Условимся далее через $C(\cdot)$ обозначать постоянные, зависящие только от параметра, стоящего в скобках, через $C$ - абсолютные постоянные, а через $\mathcal{N}(\cdot)$ - функщию распределения стандартного нормального закона.

Теорема. Пусть $\beta_{n}^{2} \neq 0, \frac{1}{3}<\gamma \leqslant \frac{2}{3}, 0<\delta \leqslant 1$. Тогда при всех $n \geqslant 2$ справедлива оченка

$$
\begin{aligned}
\Delta_{n} & =\sup _{x}\left|\mathbf{P}\left\{n \sigma_{n}^{-1} 2^{-1} U_{n}<x\right\}-\mathcal{N}(x)\right| \\
& \leqslant C(\delta)\left[\Gamma_{2+\delta}^{(n)}\right]^{2+\delta} n^{-\delta / 2}+C(\gamma)\left[\Gamma_{2}^{(n)}\right]^{2 \gamma}\left[B_{1+\gamma}^{(n)}\right]^{1+\gamma} n^{-(3 \gamma-1) / 2} .
\end{aligned}
$$

Из данной теоремы, обозначений (6)-(8) и неравенств $\nu_{p}^{(n)} \leqslant \nu_{p} \leqslant \mu_{p}$, $\mu_{p}^{(n)} \leqslant \mu_{p}, p \geqslant 2$, вытекают три следствия.

Следствие 1. Пусть $\beta_{n}^{2} \neq 0, \nu_{3}^{(n)}<\infty, \mu_{5 / 3}^{(n)}<\infty$. Тогда $n p u n \geqslant 2$ верна оченка

$$
\Delta_{n} \leqslant C \beta_{n}^{-3}\left(\nu_{3}^{(n)}+\left(\nu_{2}^{(n)}\right)^{2 / 3} \mu_{5 / 3}^{(n)}\right) n^{-1 / 2} .
$$

Следствие 2. Если $\beta^{2} \neq 0$ u $\nu_{2+\delta}<\infty, \mu_{(4+\delta) / 3}<\infty$ для какоголибо $\delta, 0<\delta \leqslant 1$, mo

$$
\Delta_{n}=O\left(n^{-\delta / 2}\right) \quad \text { npu } n \rightarrow \infty .
$$

Следствие 3. Если $\beta^{2} \neq 0 u \mu_{2+\delta}<\infty$ для некоторого $\delta, 0<\delta \leqslant 1$, то выполняется соотночение (9).

Заметим, что результаты теоремы и следствий 1-3 сохраняются и для классической модели $U$-статистик. В частности, для такой модели следствие 1 превращается (с точностью до постоянной $C$ ) в теорему 6.2.3 из [2], а следствие 3 - в теорему 6.2.4 из [2] (с $\delta \neq 0)$.

Доказательство теоремы опирается на следуюшие вспомогательные леммы.

Лемма 1. Пусть выполнены условия (1a), (1b). Тогда справедливы следующие утверждения.

$1^{\circ}$. Cтатистика $U_{n}$ представима в виде $U_{n}=2 U_{n 1}+U_{n 2}$, где $U_{n 1}, U_{n 2}$ определены равенствами (4), (5).

$2^{\circ}$. $U_{n 2}$ есть $U$-статистика с вырожденными ядрами, т.е.

$$
\mathbf{P}\left\{\mathbf{E}\left[\widetilde{h}_{i j}\left(X_{i}, X_{j}\right) \mid X_{i}\right]=0\right\}=1 \text {. }
$$

$3^{\circ}$. Для любого $p \geqslant 1$

$$
\mathbf{E}\left|\widetilde{h}_{i}\left(X_{i}\right)\right|^{p} \leqslant \nu_{p}^{(n)}, \quad \mathbf{E}\left|\tilde{h}_{i j}\left(X_{i}, X_{j}\right)\right|^{p} \leqslant 3^{p} \mu_{p}^{(n)} .
$$


$4^{\circ}$. Для любого $p, 1 \leqslant p \leqslant 2$,

$$
\mathbf{E}\left|n(n-1) U_{n 2}\right|^{p} \leqslant C(p) n^{2} \mu_{p}^{(n)} .
$$

Д ок а зате льство. Утверждения $1^{\circ}-3^{\circ}$ почти очевидны. Утверждение $4^{\circ}$ вытекает из $2^{\circ}, 3^{\circ}$ и двукратного применения теоремы [14].

Лемма 2 (см. [2, с. 205]). Для пюбого вещественного $x$ и $\alpha, 0<$ $\alpha \leqslant 1$, при всех $k=0,1,2, \ldots$ справедлива оченка

$$
\left|e^{i x}-\sum_{j=0}^{k} \frac{(i x)^{j}}{j !}\right| \leqslant 2^{1-\alpha} \alpha \prod_{j=0}^{k} \frac{1}{\alpha+j}|x|^{k+\alpha} .
$$

Д о к а з а т ел в с т в о сразу вытекает из неравенств

$$
\begin{array}{r}
\left|e^{i x}-1\right| \leqslant\left|e^{i x}-1\right|^{1-\alpha}\left|e^{i x}-1\right|^{\alpha} \leqslant 2^{1-\alpha}|x|^{\alpha}, \\
\left|e^{i x}-\sum_{j=0}^{k} \frac{(i x)^{j}}{j !}\right| \leqslant \int_{0}^{|x|}\left|e^{i x}-\sum_{j=0}^{k-1} \frac{(i x)^{j}}{j !}\right| d x, \quad k \geqslant 1 .
\end{array}
$$

Лемма 3. Пусть $\xi_{1}, \ldots, \xi_{n}$ - независимые случайные величины, $\mathbf{E} \xi_{i}=0, b_{j n}^{2}=\mathbf{D} \xi_{j}>0, d_{j n}^{2+\delta}=\mathbf{E}\left|\xi_{j}\right|^{2+\delta}<\infty, 0<\delta \leqslant 1, j=1, \ldots, n$. Пусть, далеe,

$$
\begin{aligned}
b_{n} & =\min \left\{b_{1 n}, \ldots, b_{n n}\right\}, \quad d_{n}=\max \left\{d_{1 n}, \ldots, d_{n n}\right\}, \\
B_{n}^{2} & =b_{1 n}^{2}+\cdots+b_{n n}^{2},
\end{aligned}
$$

$f(t)$ - характеристическая функция суммы $\left(\xi_{1}+\cdots+\xi_{n}\right) / B_{n}$. Тогда при условии $a_{n}=\left(d_{n} / b_{n}\right)^{2+\delta} n^{-\delta / 2} \leqslant C_{0}$ в области $D_{n}=\{t:|t| \leqslant$ $\left.C_{0} d_{n}^{-(2+\delta)} b_{n}^{2} B_{n}^{\delta}\right\}, C_{0}=2^{\delta} / 8$, справедлива оченка

$$
\left|f_{n}(t)-e^{-t^{2} / 2}\right| \leqslant \frac{4}{2^{\delta} 3} e^{-t^{2} / 3}\left(\frac{d_{n}}{b_{n}}\right)^{2+\delta} \frac{|t|^{2+\delta}}{n^{\delta / 2}} .
$$

Д о к а 3 а т е л в с т в о. Как и при получении оценок для $f_{n}(t)$ в случае $\delta=1$ (см., например, [15, гл. V, лемма 1]), запишем разложение для характеристической функции (х.ф.) $f_{j n}(t)$ величины $\xi_{j} / B_{n}$ :

$$
f_{j n}(t)=1-\frac{t^{2} b_{j n}^{2}}{2 B_{n}^{2}}+R_{j n}(t)
$$

в котором, согласно лемме 2 и неравенствам

$$
b_{n} \leqslant b_{j n} \leqslant d_{j n} \leqslant d_{n}
$$

для $R_{j n}(t)$ имеет место оценка

$$
\left|R_{j n}(t)\right| \leqslant \frac{2^{1-\delta}|t|^{2+\delta} d_{j n}^{2+\delta}}{2 B_{n}^{2+\delta}} \leqslant \frac{t^{2} b_{j n}^{2}}{2^{\delta} B_{n}^{2}}\left|\widetilde{R}_{n}(t)\right|
$$


где

$$
\widetilde{R}_{n}(t)=\frac{t^{\delta} d_{n}^{2+\delta}}{b_{n}^{2} B_{n}^{\delta}}
$$

Поскольку $|t|^{\delta} \leqslant \max \{1,|t|\}$ и, в силу (11),

$$
b_{n} \sqrt{n} \leqslant B_{n} \leqslant d_{n} \sqrt{n}
$$

то при условии $a_{n} \leqslant C_{0}=2^{\delta} / 8$ получаем при $t \in D_{n}$

$$
\begin{aligned}
\left|\widetilde{R}_{n}(t)\right| & \leqslant|t|^{\delta} a_{n}, \quad\left|\widetilde{R}_{n}(t)\right| \leqslant C_{0}, \\
\frac{t^{2} b_{j n}^{2}}{B_{n}^{2}} & \leqslant C_{0}^{2} \frac{b_{n}^{4} B_{n}^{2 \delta} d_{n}^{2}}{d_{n}^{4+2 \delta} B_{n}^{2}} \leqslant C_{0}^{2} \frac{n^{\delta} b_{n}^{2}}{n d_{n}^{2}} \leqslant C_{0}^{2} .
\end{aligned}
$$

Тогда на основании $(10),(12),(13)\left|f_{j n}(t)-1\right| \leqslant 2^{-1} C_{0}^{2}+2^{-\delta} C_{0}^{3}<\frac{1}{2}$, откуда получаем

$$
\ln f_{j n}(t)=-\frac{t^{2} b_{j n}^{2}}{B_{n}^{2}}+\widetilde{R}_{j n}(t)
$$

где с учетом (12)

$$
\begin{aligned}
\left|\widetilde{R}_{j n}(t)\right| & \leqslant\left|R_{j n}(t)\right|+2\left(\frac{t^{2} b_{j n}^{2}}{2 B_{n}^{2}}\right)^{2}+2 R_{j n}^{2}(t) \\
& \leqslant \frac{t^{2} b_{j n}^{2}}{2^{\delta} B_{n}^{2}}\left[\left|\widetilde{R}_{n}(t)\right|+\frac{t^{4} b_{j n}^{4}}{2 B_{n}^{4}}+2 \frac{t^{4} b_{j n}^{4}}{2^{2 \delta} B_{n}^{4}} \widetilde{R}_{n}^{2}(t)\right] .
\end{aligned}
$$

Из (11), (13), (14) и определения области $D_{n}$ имеем при $t \in D_{n}$

$$
\begin{aligned}
\frac{t^{2} b_{j n}^{2}}{B_{n}^{2}} & \leqslant \frac{t^{\delta} d_{n}^{2+\delta}}{b_{n}^{2} B_{n}^{\delta}}\left(C_{0} \frac{b_{n}^{2} B_{n}^{\delta}}{d_{n}^{2+\delta}}\right)^{2-\delta} \frac{b_{n}^{2} d_{n}^{2}}{d_{n}^{2+\delta} B_{n}^{2-\delta}} \\
& \leqslant C_{0}^{2-\delta}\left|\widetilde{R}_{n}(t)\right|\left(\frac{b_{n}}{d_{n}}\right)^{4-\delta} n^{(2-\delta)(1-\delta) / 2} \leqslant C_{0}\left|\widetilde{R}_{n}(t)\right| .
\end{aligned}
$$

Отсюда с помошью (15) и (17) выводим

$$
\begin{aligned}
\left|\widetilde{R}_{j n}(t)\right| & \leqslant \frac{t^{2} b_{j n}^{2}}{2^{\delta} B_{n}^{2}}\left|\widetilde{R}_{n}(t)\right|\left(1+\frac{C_{0}}{2^{1-\delta}}+2^{1-\delta} C_{0}^{4}\right) \\
& \leqslant \frac{t^{2} b_{j n}^{2}}{2^{\delta} B_{n}^{2}}\left|\widetilde{R}_{n}(t)\right|\left(1+\frac{1}{4}+\frac{1}{4^{2}}\right) \leqslant \frac{4}{3} \frac{t^{2} b_{j n}^{2}}{2^{\delta} B_{n}^{2}}\left|\widetilde{R}_{n}(t)\right| .
\end{aligned}
$$

Суммируя (16) по $j=1, \ldots, n$ и используя последнее неравенство, устанавливаем

$$
\ln f_{n}(t)=-\frac{t^{2}}{2}+R_{n}(t), \quad\left|R_{n}(t)\right| \leqslant \frac{4}{3} \frac{t^{2}}{2^{\delta}}\left|\widetilde{R}_{n}(t)\right| .
$$


Оценка (18) с учетом (15) и неравенства $\left|e^{x}-1\right| \leqslant e^{i x}|x|$, справедливого для любого комплексного $x$, приводит к утверждению леммы 3 .

Лемма 4. Для характеристической функчии $f_{n}(t)$ из лемми 3 nри $t \in D_{n}$ выполняется неравенство

$$
\left|f_{n}(t)\right| \leqslant \exp \left\{-\frac{t^{2}}{2}+\frac{|t|^{1+\delta}}{8}\right\} \text {. }
$$

Д о к а з а т е л ь т в о. Снова используем разложение (10), в котором

$$
\left|R_{j n}(t)\right| \leqslant \frac{|t|^{1+\delta} b_{j n}^{2}}{2^{\delta} B_{n}^{2}}\left|\bar{R}_{n}(t)\right|, \quad \bar{R}_{n}(t)=\frac{t d_{n}^{2+\delta}}{b_{n}^{2} B_{n}^{\delta}} .
$$

Далее, из последнего неравенства в (15), полученного без учета условия $a_{n} \leqslant C_{0}$, видно, что $t^{2} b_{j n}^{2} / B_{n}^{2}<1$, откуда в силу (10) имеем

$$
\left|f_{j n}(t)\right| \leqslant 1-\frac{t^{2} b_{j n}^{2}}{2 B_{n}^{2}}+\left|R_{j n}(t)\right| \leqslant \exp \left\{-\frac{t^{2} b_{j n}^{2}}{2 B_{n}^{2}}+\left|R_{j n}(t)\right|\right\} \text {. }
$$

Из (19) и (20) с учетом определения $B_{n}^{2}$ и $D_{n}$ вытекает, что

$$
\left|f_{n}(t)\right| \leqslant \exp \left\{-\frac{t^{2}}{2}+\left(\frac{|t|^{1+\delta}}{2^{\delta}}\right)\left|\bar{R}_{n}(t)\right|\right\}
$$

где $\left|\bar{R}_{n}(t)\right| \leqslant 2^{\delta} / 8$ при $t \in D_{n}$. Лемма 4 доказана.

Д о к а з а т л ь с т о т е о р м ы. Утверждение теоремы при $n \leqslant 8^{2 / \delta} 2^{-2}\left[\Gamma_{2+\delta}^{(n)}\right]^{2(2+\delta) / \delta}$ вытекает из тривиального неравенства $\Delta n \leqslant$ $1 \leqslant n^{-\delta / 2} 8 \cdot 2^{-\delta}\left[\Gamma_{2+\delta}^{(n)}\right]^{2+\delta}$.

Поэтому будем предполагать выполненным условие

$$
\left[\Gamma_{2+\delta}^{(n)}\right]^{2+\delta} n^{-\delta / 2} \leqslant \frac{2^{\delta}}{8}
$$

(так что при $\beta_{n}^{2} \neq 0$ в силу $(8) \nu_{2+\delta}^{(n)}<\infty$ ). Будем использовать метод доказательства теоремы 6.2 .3 из [2], основанный, в свою очередь, на идеях работы [5].

Введем обозначения

$$
\left.\begin{array}{c}
v_{j}=\sigma_{n}^{-1} \tilde{h}_{j}\left(X_{j}\right), \quad w_{j k}=\sigma_{n}^{-1}(n-1)^{-1} \tilde{h}_{j k}\left(X_{j}, X_{k}\right), \\
v(m)=\sum_{\substack{j=1 \\
v=v(n),}}^{m}, \quad w(m)=\sum_{\substack{1 \leqslant j \neq k \leqslant m \\
w=w(n) .}} w_{j k}, \\
w=
\end{array}\right\}
$$

Принимая во внимание $(4),(5),(22),(23)$ и утверждение $1^{\circ}$ леммы 1 , введем х.ф.

$$
\varphi_{n}(t)=\mathbf{E} \exp \left\{i t n \sigma_{n}^{-1} 2^{-1} U_{n}\right\}=\mathbf{E} \exp \{i t(v+w)\}
$$


Согласно известному неравенству (см., например, теорему 2 гл. V B [15]),

$$
\Delta_{n} \leqslant \int_{0}^{T_{n}} \frac{\left|\varphi_{n}(t)-e^{-t^{2} / 2}\right|}{t} d t+\frac{C}{T_{n}} .
$$

Обозначим через $J\left(T_{n}\right)$ интеграл в правой части (24) и положим

$$
T_{n}=\frac{2^{\delta} \beta_{n}^{2} \sigma_{n}^{\delta}}{8 \nu_{2+\delta}^{(n)}}
$$

где $\beta_{n}, \nu_{2+\delta}^{(n)}$ определены в (6), (7).

Тогда можем записать

$$
J\left(T_{n}\right) \leqslant \int_{0}^{T_{n}} \frac{\left|\mathbf{E} e^{i t v}-e^{-t^{2} / 2}\right|}{t} d t+\int_{0}^{T_{n}} \frac{\mathbf{E} e^{i t v}\left(e^{i t w}-1\right)}{t} d t=J_{1}+J_{2} .
$$

На основании леммы 3 , условия (21), обозначений $(6),(8)$ и (25) получаем при $t \in\left[0, T_{n}\right]$

$$
J_{1} \leqslant \frac{4}{2^{\delta} \cdot 3} \frac{\nu_{2+\delta}^{(n)}}{\beta_{n}^{2+\delta} n^{\delta / 2}} \int_{0}^{\infty} t^{1+\delta} e^{-t^{2} / 3} d t \leqslant C(\delta)\left[\Gamma_{2+\delta}^{(n)}\right]^{2+\delta} n^{-\delta / 2} .
$$

Для ощенки подынтегрального выражения в $J_{2}$ положим при $m=$ $1, \ldots, n-1$

$$
\left.\begin{array}{c}
\bar{w}_{m}=w-w(n-m), \quad \bar{v}_{m}=v-v(n-m), \\
(n-m)=\sum_{\substack{k=1, \ldots, n-m \\
k \neq j}}\left(w_{j k}+w_{k j}\right)=2 \sum_{\substack{k=1, \ldots, n-m \\
k \neq j}} w_{j k}
\end{array}\right\}
$$

и напишем

$$
\begin{aligned}
\mathbf{E} e^{i t v}\left(e^{i t w}-1\right)= & \mathbf{E} e^{i t v}\left[e^{i t w(n-m)}-1-i t w(n-m)\right] \\
& +\mathbf{E} e^{i t v} e^{i t w(n-m)}\left(e^{i t \bar{w}_{m}}-1-i t \bar{w}_{m}\right) \\
& +i t \mathbf{E} e^{i t v} w(n-m)+i t \mathbf{E} e^{i t v} e^{i t w(n-m)} \bar{w}_{m} \\
= & S_{1}(t)+S_{2}(t)+S_{3}(t)+S_{4}(t) .
\end{aligned}
$$

Согласно лемме 2 и в силу независимости величин $w(n-m)$ и $\bar{v}_{m}$,

$$
\begin{aligned}
& \left|S_{1}(t)\right| \leqslant 2^{1-\gamma} \mathbf{E}|w(n-m)|^{1+\gamma}\left|\mathbf{E} e^{i t \bar{v}_{m}}\right||t|^{1+\gamma}, \\
& \left|S_{2}(t)\right| \leqslant 2^{1-\gamma} \mathbf{E}\left|\bar{w}_{m}\right|^{1+\gamma}|t|^{1+\gamma}
\end{aligned}
$$

Далее, из $(22),(23),(28)$ с учетом симметрии $w_{j k}$ относительно $j, k$ и независимости величин $\left\{w_{j k}, v_{j}, v_{k}, w_{j l}\right\}$ и $\left\{v_{i}, i \neq j, k, l\right\}$ устанавливаем, что

$$
\left|S_{3}(t)\right| \leqslant|t| \sum_{1 \leqslant i \neq k \leqslant n-m},\left|\mathbf{E} w_{j k} e^{i t v_{j}} e^{i t v_{k}}\right|\left|\mathbf{E} e^{i t\left(v-v_{j}-v_{k}\right)}\right|
$$




$$
\begin{aligned}
\left|S_{4}(t)\right| \leqslant & |t| \sum_{n-m \leqslant j \neq k \leqslant n}\left|\mathbf{E} w_{j k} e^{i t v_{j}} e^{i t v_{k}}\right|\left|\mathbf{E} e^{i t\left(\bar{v}_{m}-v_{j}-v_{k}\right)}\right| \\
& +2|t| \sum_{j=1}^{n-m} \sum_{k=n-m+1}^{n} \mid \mathbf{E} w_{j k} e^{i t v_{j}} e^{i t v_{k}} e^{i t \bar{w}_{j}} \\
& \times \exp \left\{i t\left[v(n-m)+w(n-m)-v_{j}-w_{j}(n-m)\right]\right\} \mid \\
& \times\left|\mathbf{E} e^{i t\left(\bar{v}_{m}-v_{k}\right)}\right|=S_{41}(t)+S_{42}(t) .
\end{aligned}
$$

Введем обозначения

$$
\begin{aligned}
a_{n}(t) & =\sum_{1 \leqslant i \neq j \leqslant n} \mathbf{E}\left|w_{j k}\left(e^{i t v_{j}}-1\right)\left(e^{i t v_{k}}-1\right)\right| \\
b_{n}(t) & =\sum_{i=1}^{n-m} \sum_{k=n-m+1}^{n} \mathbf{E}\left|w_{j k}\left(e^{i t w_{j}(n-m)}-1\right)\left(e^{i t v_{k}}-1\right)\right| \\
C_{n}(t) & =\max _{1 \leqslant l \leqslant n}\left|\mathbf{E} e^{i t v_{l}}-1\right| .
\end{aligned}
$$

Поскольку в силу (22) и утверждения $2^{\circ}$ леммы 1 для любой функции $f(\cdot)$ при $i_{1} \neq i_{2}$

$$
\mathrm{E} w_{i_{1} i_{2}} f\left(v_{i_{3}}, w_{i_{4} i_{5}}, i_{3}, i_{4}, i_{5}=1, \ldots, n, i_{3}, i_{4}, i_{5} \neq i_{1}\right)=0
$$

то из $(32)-(36)$ и независимости величин $\left\{v_{j}\right\}$ вытекақо неравенства

$$
\begin{aligned}
\left|S_{3}(t)\right| & \leqslant|t| a_{n}(t)\left[1-C_{n}(t)\right]^{-2}\left|\mathbf{E} e^{i t v}\right| \\
\left|S_{41}(t)\right| & \leqslant|t| a_{n}(t)\left[1-C_{n}(t)\right]^{-2}\left|\mathbf{E} e^{i t \bar{v}_{m}}\right| \\
\left|S_{42}(t)\right| & \leqslant|t|\left[a_{n}(t)+2 b_{n}(t)\right]\left[1-C_{n}(t)\right]^{-1}\left|\mathbf{E} e^{i t \bar{v}_{m}}\right|
\end{aligned}
$$

Для дальнейших оценок величин $S_{1}(t)-S_{4}(t)$ нам понадобится ряд вспомогательных предложений.

Предложение 1. Для любыл $q, p \geqslant 1$

(a) $\mathrm{E}\left|v_{j}\right|^{q} \leqslant \sigma_{n}^{-q} \nu_{q}^{(n)}(n) ;$ (b) $\mathrm{E}\left|w_{j k}\right|^{p} \leqslant 6^{-p} \sigma_{n}^{-p} n^{-p} \mu_{p}^{(n)}$.

Это вытекает из (22) и утверждения $3^{\circ}$ леммы 1 .

Предложение 2. Дия пюбых $p, 1<p \leqslant 2, u m=1, \ldots, n-1$, $j=1, \ldots, n$ справедливы неравенства

(a) $\mathbf{E}|w(n-m)|^{p} \leqslant C(p) \sigma_{n}^{-p} n^{-p+2} \mu_{p}^{(n)}$

(b) $\mathbf{E}\left|\bar{w}_{m}\right|^{p} \leqslant C(p) \sigma_{n}^{-p} n^{-p+1} m \mu_{p}^{(n)}$,

(c) $\mathbf{E}\left|w_{j}(n-m)\right|^{p} \leqslant C(p) \sigma_{n}^{-p} n^{-p+1} \mu_{p}^{(n)}$.

Здесь (a) непосредственно следует из утверждения $4^{\circ}$ леммы 1 , примененного к статистике $U_{n-m}$, a (b) и (c) выводятся аналогично.

Прредложение 3. Для любых $i=1, \ldots, n, q>2$ верны неравенства (a) $\beta_{n} \leqslant \beta_{i n} \leqslant \sqrt{\nu_{2}^{(n)}} \leqslant\left(\nu_{q}^{(n)}\right)^{1 / q} ;$ (b) $1 \leqslant \Gamma_{2}^{(n)} \leqslant \Gamma_{q}^{(n)}$. 
Эти неравенства вытекают из (4), (6)-(8) и утверждения $3^{\circ}$ леммы 1.

Предложение 4. Для любы $m=1, \ldots, n-1, n \geqslant 2$

(a) $\beta_{n}^{2} n \leqslant \sigma_{n}^{2} \leqslant \nu_{2}^{(n)}(n)$; (b) $\beta_{n}^{2} m \leqslant \sigma_{n}^{2}-\sigma_{n-m}^{2}$.

Это следует из (6) и.предложения $3(\mathrm{a})$.

Предложение 5. При $0<\delta<1, \frac{1}{3}<\gamma \leqslant \frac{2}{3}$ для величины (34) справедлива оченка

$$
\left|a_{n}(t)\right| \leqslant \frac{2}{3}\left[\frac{\nu_{2+\delta}^{(n)} n}{\sigma_{n}^{2+\delta}}|t|^{2+\delta}+\frac{\left(\nu_{2}^{(n)}\right)^{\gamma} \mu_{1+\gamma}^{(n)} n}{\sigma_{n}^{3 \gamma+1}}|t|^{2 \gamma}\right]
$$

Доказ ат ль ьство. Пусть

$$
\begin{aligned}
& \alpha=\min \left\{(2+\delta) \gamma(1+\gamma)^{-1}, 1\right\}, \\
& \bar{\alpha}=\max \left\{0,(2+\delta) \gamma(1+\gamma)^{-1}-1\right\}, \quad \bar{\gamma}=2 \gamma(1+\gamma)^{-1}
\end{aligned}
$$

$J_{j k}$ - индикаторная функция множества $\left\{v_{k} \leqslant v_{j}\right\}$. Учитывая, что

$$
\alpha+\bar{\alpha}=(2+\delta) \gamma(1+\gamma)^{-1}, \quad \bar{\alpha}+\bar{\gamma} \leqslant 1, \quad \alpha+\bar{\alpha}+\bar{\gamma} \geqslant 1, \quad 1-J_{j k} \leqslant J_{k j}
$$

получаем в силу леммы 2

$$
\begin{aligned}
& \mathbf{E}\left|w_{j k}\left(e^{i t v_{j}}-1\right)\left(e^{i t v_{k}}-1\right)\right| \\
& \leqslant 2^{1-\alpha} 2^{1-\bar{\alpha}-\bar{\gamma}} \mathbf{E}\left|w_{j k}\right|\left[\left|t v_{j}\right|^{\alpha}\left|t v_{k}\right|^{\bar{\alpha}+\bar{\gamma}} J_{j k}+\left|t v_{j}\right|^{\bar{\alpha}+\bar{\gamma}}\left|t v_{k}\right|^{\alpha} J_{k j}\right] \\
& \leqslant 2|t|^{[(2+\delta) \gamma+2 \gamma] / 1+\gamma} \mathbf{E}\left|w_{j k}\right| \\
& \times\left[\left|v_{j}\right|^{(2+\delta) \gamma /(1+\gamma)}\left|v_{k}\right|^{2 \gamma /(1+\gamma)}+\left|v_{j}\right|^{2 \gamma /(1+\gamma)}\left|v_{k}\right|^{(2+\delta) \gamma /(1+\gamma)}\right] .
\end{aligned}
$$

Отсюда, пользуясь неравенством Гёльдера и независимостью величин $v_{j}, v_{k}$ при $j=k$, получаем

$$
\begin{aligned}
& \mathbf{E}\left|w_{j k}\left(e^{i t v_{j}}-1\right)\left(e^{i t v_{k}}-1\right)\right| \leqslant 2|t|^{[(2+\delta) \gamma+2 \gamma] /(1+\gamma)}\left(\mathbf{E}\left|w_{j k}\right|^{1+\gamma}\right)^{1 /(1+\gamma)} \\
& \times\left[\left(\mathbf{E}\left|v_{j}\right|^{2+\delta} \mathbf{E} v_{k}^{2}\right)^{\gamma /(1+\gamma)}+\left(\mathbf{E} v_{j}^{2} \mathbf{E}\left|v_{k}\right|^{2+\delta}\right)^{\gamma /(1+\gamma)}\right]
\end{aligned}
$$

Из (34), (40) и предложений 1(a), (b) выводим

$$
\begin{aligned}
\left|a_{n}(t)\right| \leqslant & \frac{2}{3}|t|^{[(2+\delta) \gamma+2 \gamma] /(1+\gamma)}\left[\nu_{2+\delta}^{(n)}\right]^{\gamma /(1+\gamma)} \\
& \times\left(\nu_{2}^{(n)}\right)^{\gamma /(1+\gamma)}\left[\mu_{1+\gamma}^{(n)}\right]^{1 /(1+\gamma)}\left(\frac{n}{\sigma_{n}^{2+\delta}}\right)^{\gamma /(1+\gamma)}\left(\frac{n}{\sigma_{n}^{3 \gamma+1}}\right)^{1 /(1+\gamma)} .
\end{aligned}
$$

Последняя оценка и неравенство $|a b| \leqslant|a|^{p}+|b|^{q}$, справедливое при $p, q>1,1 / p+1 / q=1$, доказывают предложение 5 .

Предложение 6. При $0<\gamma \leqslant 1$ для величин (35) верна оченка

$$
\left|b_{n}(t)\right| \leqslant C(\gamma)|t|^{\left(3 \gamma+\gamma^{2}\right) /(1+\gamma)} \mu_{1+\gamma}^{(n)}\left[\nu_{2}^{(n)}\right]^{2 \gamma /(1+\gamma)} \frac{n^{1-\gamma}}{\sigma_{m}^{1+\gamma}}\left(\frac{n}{\sigma_{m}^{2}}\right)^{\gamma /(1+\gamma)} .
$$


Д о к а з а т л л с т в о. Применяя лемму 2, а затем неравенство Гёльдера, с учетом (28) и независимости величин $w_{j}(n-m), v_{k}$ при $j \leqslant$ $n-m, k>n-m$ получаем

$$
\begin{aligned}
& \mathbf{E}\left|w_{j k}\left(e^{i t w_{j}(n-m)}-1\right)\left(e^{i t v_{k}}-1\right)\right| \\
& \leqslant 2^{1-\gamma} 2^{1-2 \gamma /(1+\gamma)}|t|^{\gamma+2 \gamma /(1+\gamma)} \mathbf{E}\left|w_{j k}\right|\left|w_{j}(n-m)\right|^{\gamma}\left|v_{k}\right|^{2 \gamma /(1+\gamma)} \\
& \leqslant 2 \sqrt{2}|t|^{\left(3 \gamma+\gamma^{2}\right) /(1+\gamma)}\left(\mathbf{E}\left|w_{j k}\right|^{1+\gamma}\right)^{1 /(1+\gamma)} \\
& \times\left(\mathbf{E}\left|w_{j}(n-m)\right|^{1+\gamma}\right)^{\gamma /(1+\gamma)}\left(\mathbf{E} v_{k}^{2}\right)^{\gamma /(1+\gamma)}
\end{aligned}
$$

Соотношения (35), (41) и предложения $1(\mathrm{a}), 1(\mathrm{~b}), 2(\mathrm{~b})$ доказывают предложение 6 .

Предложение 7. При $\beta_{n}^{2} \neq 0, \nu_{2+\delta}^{(n)}<\infty, 0<\delta \leqslant 1, t \in\left[0, T_{n}\right]$ величина (36) удовлетворяет неравенству $\left|C_{n}(t)\right| \leqslant \frac{1}{4}$.

Д о к а 3 а т е л ь с т в о вытекает из соотношения

$$
\left|C_{n}(t)\right| \leqslant \max _{1 \leqslant l \leqslant n} T_{n} \sqrt{\mathbf{E} v_{l}^{2}}
$$

обозначения (25) и предложений $1(\mathrm{a}), 3(\mathrm{a})$.

Предложение 8. Пусть $\beta_{n}^{2} \neq 0$, выполнено условие (21) $u$

$$
m=\min \left\{n-1, \sigma_{n}^{2} \beta_{n}^{-2}|t|^{-(1+\gamma+\varepsilon)}+1\right\}, \quad \varepsilon=\frac{1-\gamma}{2} .
$$

Тогда при $t \in\left[0, T_{n}\right]$ верны оценки

(a) $\left|\mathbf{E} e^{i t v}\right| \leqslant e^{1 / 8} e^{-3 t^{2} / 8}$

(b) $\left|\mathbf{E} e^{i t \bar{v}_{m}}\right| \leqslant e^{1 / 8}\left(e^{-9 t^{2} / 32}+e^{-3 t^{t} / 8}\right)$.

Д о к а з а т е л с т в о. Оценка (а) следует из леммы 4, обозначений $(6),(22),(23),(25)$, предложения 1(а) и неравенства

$$
|t|^{1+\delta} \leqslant \max \left\{1, t^{2}\right\} \text {. }
$$

Далее, обозначим через $\Psi_{m}(\cdot)$ х.ф. величины $\bar{\sigma}_{m}^{-1} \sum_{i=n-m+1}^{n} \widetilde{h}_{i}\left(X_{i}\right)$, где $\bar{\sigma}_{m}^{2}=\mathbf{D}\left[\sum_{i=n-m+1}^{n} \tilde{h}_{i}\left(X_{i}\right)\right]$. Нетрудно видеть, что, согласно (6), $(22),(28)$,

$$
\mathbf{E} e^{i t \bar{v}_{m}}=\psi_{m}\left(\tau_{n}\right), \quad \tau_{n}=\frac{t \sqrt{\sigma_{n}^{2}-\sigma_{n-m}^{2}}}{\sigma_{n}},
$$

и, кроме того, в силу (6), (25) и утверждения $3^{\circ}$ леммы 1

$$
0<\tau_{n} \leqslant \frac{2^{\delta}}{8} \frac{\bar{\beta}_{n}^{2}}{\nu_{2+\delta}^{(n)}} \bar{\sigma}_{m}^{\delta}\left(\frac{\bar{\sigma}_{m}}{\sigma_{n}}\right)^{1-\delta} \leqslant \frac{2^{\delta} \bar{\beta}_{m}^{2}}{\bar{\nu}_{2+\delta}^{(n)}} \bar{\sigma}_{m}^{\delta}
$$

где $\bar{\beta}_{m}^{2}=\min \mathbf{D} \widetilde{h}_{i}\left(X_{i}\right), \bar{v}_{2+\delta}^{(m)}=\max \mathbf{E}\left|\tilde{h}_{i}\left(X_{i}\right)\right|^{2+\delta}$, а $\min$ и $\max$ берутся по $i=n-m+1, \ldots, n$. 
Тогда к х.ф. $\psi_{m}\left(\tau_{n}\right)$ можно применить оценку (а), откуда следует, что

$$
\left|\psi_{m}\left(\tau_{n}\right)\right| \leqslant e^{1 / 8} e^{-3 \tau_{n}^{2} / 8}
$$

или же, на основании (43),

$$
\left|\mathbf{E} e^{i t \bar{v}_{m}}\right| \leqslant e^{1 / 8} \exp \left\{-\frac{3}{8} t^{2} \frac{\sigma_{n}^{2}-\sigma_{n-m}^{2}}{\sigma_{n}^{2}}\right\}
$$

Теперь заметим, что при $m=n-1$ благодаря предложениям $3(\mathrm{a}), 4(\mathrm{a})$

$$
\left(\sigma_{n}^{2}-\sigma_{n-m}^{2}\right) \sigma_{m}^{-2}=1-\beta_{1 n}^{2} \sigma_{n}^{-2} \geqslant 1-\frac{\left(\nu_{2+\delta}^{(n)}\right)^{2 /(2+\delta)}}{\beta_{n}^{2} n},
$$

а при $m=\left[\sigma_{n}^{2} \beta_{n}^{-2}|t|^{-(1+\gamma+\varepsilon)}\right]+1$ в силу предложения $4(\mathrm{~b})$

$$
\left(\sigma_{n}^{2}-\sigma_{n-m}^{2}\right) \sigma_{n}^{-2} \geqslant \beta_{n}^{2} m \sigma_{n}^{-2} \geqslant|t|^{-(1+\gamma+\varepsilon)} \text {. }
$$

Последние два неравенства с учетом (8) и условия (21) дают

$$
\left(\sigma_{n}^{2}-\sigma_{n-m}^{2}\right) \sigma_{m}^{-2} \geqslant \min \left\{\frac{3}{4},|t|^{-(1+\gamma+\varepsilon)}\right\} \text {. }
$$

Из неравенств (44), (45) и равенства $\varepsilon=(1-\gamma) / 2$ следует оценка (b). ПІредложение 8 доказано.

Перейдем к получению окончательных оценок для слагаемых из правой части (29), всюду пользуясь обозначениями (8) и предполагая, что выполнено условие $(21), t \in\left[0, T_{n}\right]$ и $m$ определяется равенством (42). В силу (30) и предложений $2(\mathrm{a}), 4(\mathrm{a}), 8(\mathrm{~b})$,

$$
\begin{aligned}
\left|S_{1}(t)\right| & \leqslant C(\gamma) \sigma_{n}^{-(1+\gamma)} n^{1-\gamma} \mu_{1+\gamma}^{(n)} t^{1+\gamma}\left(e^{-9 t^{2} / 32}+e^{-3 t^{\varepsilon} / 8}\right) \\
& \leqslant C(\gamma)\left[B_{1+\gamma}^{(n)}\right]^{1+\gamma} n^{-(3 \gamma-1) / 2} t^{1+\gamma}\left(e^{-9 t^{2} / 32}+e^{-3 t^{\varepsilon} / 8}\right) .
\end{aligned}
$$

Далее, из (31) и предложения 2(b)

$$
\begin{aligned}
\left|S_{2}(t)\right| \leqslant & C(\gamma) \sigma_{n}^{-(1+\gamma)} n^{1-\gamma} \mu_{1+\gamma}^{(n)} t^{1+\gamma} \\
& \times \min \left\{\frac{n-1}{n}, \frac{\sigma_{n}^{2} \beta_{n}^{-2} t^{-(1+\gamma+\varepsilon)}+1}{n}\right\} .
\end{aligned}
$$

Поскольку в силу (25), предложения 4(а) и условия (21) $1 \leqslant T_{n} \leqslant \sqrt{n}$ и, значит, $\sigma_{n}^{2} \beta_{n}^{-2} t^{-(1+\gamma+\varepsilon)} \geqslant n T_{n}^{-2} \geqslant 1$, то, согласно (47)

$$
\left|S_{2}(t)\right| \leqslant C(\gamma) \sigma_{n}^{-(1+\gamma)} n^{1-\gamma} \mu_{1+\gamma}^{(n)} t^{1+\gamma} \min \left\{1, \frac{2 \sigma_{n}^{2} \beta_{n}^{-2} t^{-(1+\gamma+\varepsilon)}}{n}\right\}
$$


- Отсюда, применяя нижнюю и верхнюю оценки для $\sigma_{n}$ в предложении $4(\mathrm{a})$, устанавливаем

$$
\left|S_{2}(t)\right| \leqslant C(\gamma)\left[B_{1+\gamma}^{(n)}\right]^{1+\gamma}\left(1+\left[\Gamma_{2}^{(n)}\right]^{1-\gamma}\right) \min \left\{t^{1+\gamma}, t^{-\varepsilon}\right\}
$$

Теперь на основании неравенства (37) и предложений $5,7,8(\mathrm{a})$ получаем

$$
\begin{aligned}
\left|S_{3}(t)\right| \leqslant C( & {\left[\Gamma_{2+\delta}^{(n)}\right]^{2+\delta} n^{-\delta / 2} t^{3-\delta} } \\
& \left.+\left[\Gamma_{2}^{(n)}\right]^{2 \gamma}\left[B_{1+\gamma}^{(n)}\right]^{1+\gamma} n^{-(3 \gamma-1) / 2} t^{1+2 \gamma}\right) e^{-3 t^{2} / 8} .
\end{aligned}
$$

Наконец, из (33), (38), (39), и предложений 5-7 и 8(b) выводим

$$
\begin{aligned}
\left|S_{4}(t)\right| \leqslant\{ & C\left(\left[\Gamma_{2+\delta}^{(n)}\right]^{2+\delta} n^{-\delta / 2} t^{3+\delta}+\left[\Gamma_{2}^{(n)}\right]^{2 \gamma}\right. \\
& \left.\times\left[B_{1+\gamma}^{(n)}\right]^{1+\gamma} n^{-(3 \gamma-1) / 2} t^{1+2 \gamma}\right) \\
+ & C(\gamma)\left[\Gamma_{2}^{(n)}\right]^{2 \gamma /(1+\gamma)}\left[B_{1+\gamma}^{(n)}\right]^{1+\gamma} \\
& \left.\times n^{-(3 \gamma-1) / 2} t^{1+\left(3 \gamma+\gamma^{2}\right) /(1+\gamma)}\right\}\left(e^{-9 t^{2} / 16}+e^{-3 t^{\varepsilon} / 8}\right)
\end{aligned}
$$

Вернемся к интегралу $J_{2}$ из неравенства (26). Согласно (29),

$$
J_{2} \leqslant \sum_{i=1}^{4} \int_{0}^{T_{n}} \frac{1}{t}\left|S_{i}(t)\right| d t
$$

откуда на основании оценок (46), (48)-(50) и неравенства

$$
\max \left\{1,\left[\Gamma_{2}^{(n)}\right]^{1-\gamma},\left[\Gamma_{2}^{(n)}\right]^{2 \gamma /(1+\gamma)}\right\} \leqslant\left[\Gamma_{2}^{(n)}\right]^{2 \gamma}
$$

при $\frac{1}{3}<\gamma \leqslant \frac{2}{3}$, вытекающего из предложения $3(\mathrm{~b})$, получаем

$$
J_{2} \leqslant C(\delta)\left[\Gamma_{2+\delta}^{(n)}\right]^{2+\delta} n^{-\delta / 2}+C(\gamma)\left[\Gamma_{2}^{(n)}\right]^{2 \gamma}\left[B_{1+\gamma}^{(n)}\right]^{1+\gamma} n^{-(3 \gamma-1) / 2}
$$

Заметим еше, что в силу (8), (25) и предложения 4(a)

$$
T_{n} \geqslant \frac{n^{\delta / 2}}{\left[\Gamma_{2+\delta}^{(n)}\right]^{2+\delta}} .
$$

Соотношения $(24),(26),(27),(51),(52)$ завершают доказательство теоремы.

Ав'торы благодарны рецензенту за ряд полезных замечаний. 


\section{СПИСОК ЛИТЕРАТУРЫ}

1. Hoeffding $W$. A class of statistics with asymptotically normal distribution. - Ann. Math. Statist., 1948, v. 19, p. 293-325. (Перев. на русск. яз.: Сб. перев. «Математика», 1958, т. 2, № 3, c. 129-158.)

2. Королюк В. С., Боровских Ю. В. Теория $U$-статистик. Киев: Наукова думка, $1989,383 \mathrm{c}$.

3. Hsy $P$. Z. The approximate distribution of mean and variance of a sample of independent variables. - Ann. Math. Statist., 1945, v. 16, № 1, p. 1-29.

4. Bickel P. J. Edgeworth expansions in non-parametric statistics. - Ann. Math. Statist., 1974, v. 2, № 1, p. 1-20.

5. Chen Y. K., Wierman T. On the Berry-Esseen theorem for $U$-statistics. - Ann. Probab., 1977, v. 5, № 1, p. 136-139.

6. Callaert $H$., Janssen $P$. The Berry-Esseen theorem for $U$-statistics. - Ann. Statist., 1978 , v. 6 , № 2, p. 417-421.

7. Малевич T. Л., Абдалимов Б. Об оценке уклонения распределения $U$-статистики от нормального распределения. - Изв. АН У c. 10-13.

8. Михайлов $B$. Г. Центральная предельная теорема для неоднородных $U$-статистик от конечно-зависимых случайных величин. - Матем. сб., 1975, т. 98, № 4, с. 624634.

9. Гирко В. П. Предельные теоремы для функций случайных величин. Киев: Вища школа, 1983, $207 \mathrm{c.}$

10. Хашимов III. A. Предельные теоремы для обобщенных $U$-статистик и их статистические применения. - Докл. АН У

11. Малевич Т. Л., Абдурахманов $Г$. Р. Центральная предельная́ теорема для обобщенных (неоднородных) $U$-статистик от различно распределенных случайных величин. - Изв. АН УзССР, сер. физ.-мат. наук, 1986, № 2, с. 28-33.

12. Малевич Т. Л., Абдурахманов $Г$. Р. Необходимые и достаточные условия сходимости к безгранично делимым законам для обобщенных $U$-статистик. - Узб. матем. журн. 1991, № 5, с. 30-37.

13. Абдурахманов $Г$. $Р$. Равномерная оценка в центральной предельной теореме для обобщенных $U$-статистик. Деп. в ВИНИТИ, Ташкент, 1985, № 3179-85, 24 с.

14. Bahr B. von, Esseen C. G. Inequalities for the $r$-th absolute moment of a sum of random variables. - Ann. Math. Statist., 1965, v. 36, p. 299-303.

15. Петров В. В. Суммы независимых случайных величин. М.: Наука, 1974, 414 с.

Поступила в редакцию 21.V.1996 